\title{
Cultural adaptation of cognitive behavioural therapy (CBT) for patients with depression and anxiety in Saudi Arabia and Bahrain: a qualitative study exploring views of patients, carers, and mental health professionals
}

\author{
Haifa Mohammad Saleh Algahtani ${ }^{1 \star \star}$, Abdullah Almulhim ${ }^{2}$, Fatema Ali AlNajjar ${ }^{1}$, \\ Mazen Khalil Ali ${ }^{1}$, Muhammad $\operatorname{Irfan}^{3}$ (D), Muhammad Ayub ${ }^{4}$ and Farooq Naeem ${ }^{5}$ \\ ${ }^{1}$ College of Medicine \& Medical Sciences, Psychiatry Department, Arabian Gulf University, Bahrain, ${ }^{2}$ Psychiatry Department, \\ Imam Abdulrahman Bin Faisal University, Saudi Arabia, ${ }^{3}$ Department of Mental Health, Psychiatry \& Behavioural Sciences, \\ Peshawar Medical College, Riphah International University, Islamabad, Pakistan, ${ }^{4}$ Division of Developmental Disabilities, \\ Department of Psychiatry, Queen's University, 191 Portsmouth Avenue, Kingston, Ontario, Canada and ${ }^{5}$ Psychiatry \\ Department, University of Toronto and Schizophrenia Program, Centre for Addiction and Mental Health, Toronto, Canada \\ ${ }^{*}$ Corresponding author. Email: haifamsg@agu.edu.bh
}

(Received 27 October 2018; revised 25 June 2019; accepted 10 July 2019)

\begin{abstract}
Western values influence cognitive behavioural therapy (CBT) as it was primarily developed and practised in the West. As understanding the cultural context has been linked to better therapy outcomes, it has been suggested that CBT might need modification to non-Western clients' cultural backgrounds. Previously we developed a cost-effective approach to adapt CBT for clients in China and Pakistan. In this study, we applied the same methodology for local clients suffering from depression and anxiety in the Kingdom of Saudi Arabia and Bahrain. This study aimed to understand the views of patients with depression and anxiety, caregivers and mental health professionals about CBT to develop guidelines for culturally adapting CBT for depression and anxiety. We conducted semi-structured interviews with the patients $(n=42)$, caregivers $(n=11)$, and psychiatrists and psychologists $(n=16)$. The data were analysed using a thematic framework analysis by identifying emerging themes and categories. The themes emerging from the analyses of interviews by each interviewer were compared and contrasted with those of other interviewers. The results highlighted barriers of access to and strengths of CBT while working with these patient groups. Patients and their caregivers in both countries use a bio-psycho-spiritual-social model of illness and seek help from multiple sources. Therapists emphasized the need for using local idioms, culturally appropriate translation and minor adjustments in therapy. There were no thematic differences between the two sites. These findings will be used to culturally adapt a CBT manual, which will be tested in a randomized controlled trial.
\end{abstract}

\section{Key learning aims}

After reading this article, readers will be able to:

(1) Understand the need for cultural adaptation of CBT.

(2) Identify the necessary steps to adapt CBT for the Muslim Arab population.

(3) Understand the modifications required to deliver culturally adapted CBT for the Muslim Arab population.

Keywords: Arab Muslim; adaptation; CBT; cognitive therapy; culture; qualitative 


\section{Introduction}

There is evidence to suggest that cognitive behaviour therapy (CBT) can be useful in treating mental health problems in non-Western countries, despite its limited availability in these countries. CBT was critiqued for being value-laden (Hays and Iwamasa, 2006). Early researchers (Scorzelli and Reinke-Scorzelli, 1994) have asserted that the values underpinning CBT might conflict with the cultural values and beliefs of individuals from non-Western cultures. Explanatory models of illness are often rooted in local cultural and/or religious beliefs and values which in turn influence the help-seeking and health-related behaviours (Draine and Solomon, 1994; Joel et al., 2003; Kleinman, 1980; Lazare et al., 1975; Littlewood, 1990; Naeem et al., 2010b; Naeem et al., 2016; Rhermoul et al., 2017; Weiss, 1997). Even health workers hold indigenous beliefs about mental illness which differ markedly from the biopsychosocial models emphasized by mental health professionals and focus mostly on biological explanation (Joel et al., 2003). Understanding the cultural context and influences when applying psychotherapy is likely to lead to better outcomes (Gaytandjieva and Bontcheva, 2013).

Cultural adaptation in the treatment context includes modification of specific protocols to become culturally compatible with clients' meanings and values. Therapists working with ethnic minority patients in the USA have highlighted the core principles for adapting therapeutic techniques (Bernal et al., 1995; Bernal et al., 2009; Hwang, 2006; Tseng, 2004)

Most of these studies describe therapists' personal experiences of working with Chinese or Latino patients and address broader therapeutic issues rather than issues about a particular modality of therapy. Furthermore, these studies do not directly examine outcomes resulting from adapting therapeutic techniques to address cultural issues in therapy.

This, however, is an emerging field. Literature comparing culturally adapted versus unadapted CBT is inconclusive. One pilot study by Kohn et al. (2002) found culturally adapted CBT to be better than the unadapted CBT, while another by Hwang et al. (2015) did not find a difference between the two.

A recent review of metanalyses further highlights both the pitfalls and promises in this area (Rathod et al., 2018). Studies in Pakistan, England and China using mixed methods have revealed that numerous adjustments need to be made for CBT to be acceptable, accessible and useful for clients from non-Western cultures, taking into consideration cultural and religious or spiritual factors, service structure, delivery and resources, and patient's knowledge and beliefs about health, illness and treatment. Therefore, it has been recommended that CBT needs cultural adaptation before its application in non-Western cultures (Bernal et al., 1995; Hays and Iwamasa, 2006; Iwamasa, 1993; Naeem et al., 2010b). Existence of numerous models of cultural adaptation testifies to the importance and complexity of the adaptation process (Domenech Rodríguez et al., 2010)

A recently published meta-analysis of CBT for an Arab adult population experiencing depression, anxiety and post-traumatic stress disorder evaluated nine studies, of which only three studies reported randomized controlled trials (RCTs) (Kayrouz et al., 2018). CBT has become popular in recent years in the Kingdom of Saudi Arabia (KSA) and Bahrain (BAH), and people specifically seek this treatment in private and public health sectors. At present, there are two CBT diploma-training programmes in KSA. Professionals completing such training are far from meeting the increased demand (Algahtani et al., 2017).

In this study, we chose to use an adaptation framework based on what was initially developed using indepth qualitative interviews by the experts (Naeem et al., 2009a; Naeem et al., 2009b; Naeem et al., 2010b; Naeem et al., 2011; Naeem et al., 2012). However, such adaptation work required expertise and resources which were not readily available to us. We therefore developed semi-structured interview guides, and established areas of adaptation that can be used with relatively few resources. This modified method has been used in Pakistan and China to adapt CBT for psychosis (Li et al., 2017; Naeem et al., 2014) and to explore the views of depressed women, their carers and professionals in Morroco (Rhermoul et al., 2017). 


\section{Study aims and objectives}

The overall aim of this study was to gather information from the stakeholders (patients, carers and mental health providers) that could be used to culturally adapt CBT for depression and anxiety in KSA and BAH. Therefore, our objectives were: (a) to explore the patient's beliefs about depression and anxiety, its causes and its treatment, especially their experience of any non-pharmacological help such as CBT they may have received; (b) to gain an understanding of caregivers views about depression and anxiety, its causes and treatment; and (c) to explore the experience of psychologists and psychiatrists in KSA and BAH who treat patients with depression and anxiety, the barriers they have to overcome in treating these patients, and which CBT techniques were useful or require modification.

\section{Method}

\section{Study design and setting}

This qualitative study consisted mainly of one-to-one semi-structured interviews with patients, their caregivers, psychologists and psychiatrists. Semi-structured interviews with open-ended questions, prompts and facilitatory statements were considered to be the most suitable techniques for this study, as they give the researcher more control over the topics of the interview than in unstructured interviews, but without limiting the range of responses to each question as is the case in structured interviews or questionnaires that use closed questions (Kvale, 1996). The use of semi-structured interviews enables flexible data collection, providing the opportunity to elicit open responses while ensuring relevant topics were covered in each interview (Kvale and Brinkmann, 2009). They are also cost-effective in that they may be conducted by less experienced interviewers under supervision.

After obtaining IRB approval, this evaluation was carried out at King Fahd Hospital of the University (KFHU), Imam Abdulrahman Bin Faisal Universityin AlKhobar, KSA, and Salmaniyah Psychiatric Hospital in Manama, BAH. The former is the main teaching hospital in the Eastern Region of KSA for medical students and residents in different specialties; it consists of 440 beds of which 24 are designated for psychiatry. In addition to inpatient services, KFHU provides outpatient services with psychotherapy, consultation-liaison and day-treatment. The Salmaniyah Psychiatric Hospital is the only psychiatric hospital in BAH; it has 260 inpatient beds as well as outpatient, consultation-liaison and community psychiatry services.

\section{Sample recruitment}

A number of 'key informants' were purposively sampled for their knowledge and experience of assessing, treatment planning, and caring for this group of patients. We based our choice of groups of informants on our previous successful work in this area. Additionally, we employed the maximum variation strategy, a purposive sampling method that seeks to maximize sample heterogeneity to capture a breadth of views and perspectives (Creswell, 2009). Data saturation, the point qualitative researchers refer to at which additional data collection neither adds new information nor requires revisions to be made to findings already developed, was estimated to be achieved after approximately 30-35 interviews (Morse and Field, 1996). However, in practice, the themes saturated with a smaller number.

We first made a list of likely participants from our knowledge. They were then contacted via email invitation and followed up by telephone. Those who consented were interviewed.

\section{Development of semi-structured interviews}

Semi-structured interviews were used for this study. In our initial work in Pakistan to culturally adapt CBT for depression, open-ended interviews were conducted by a psychiatrist trained in CBT 
Table 1. Summary of themes explored through interviews

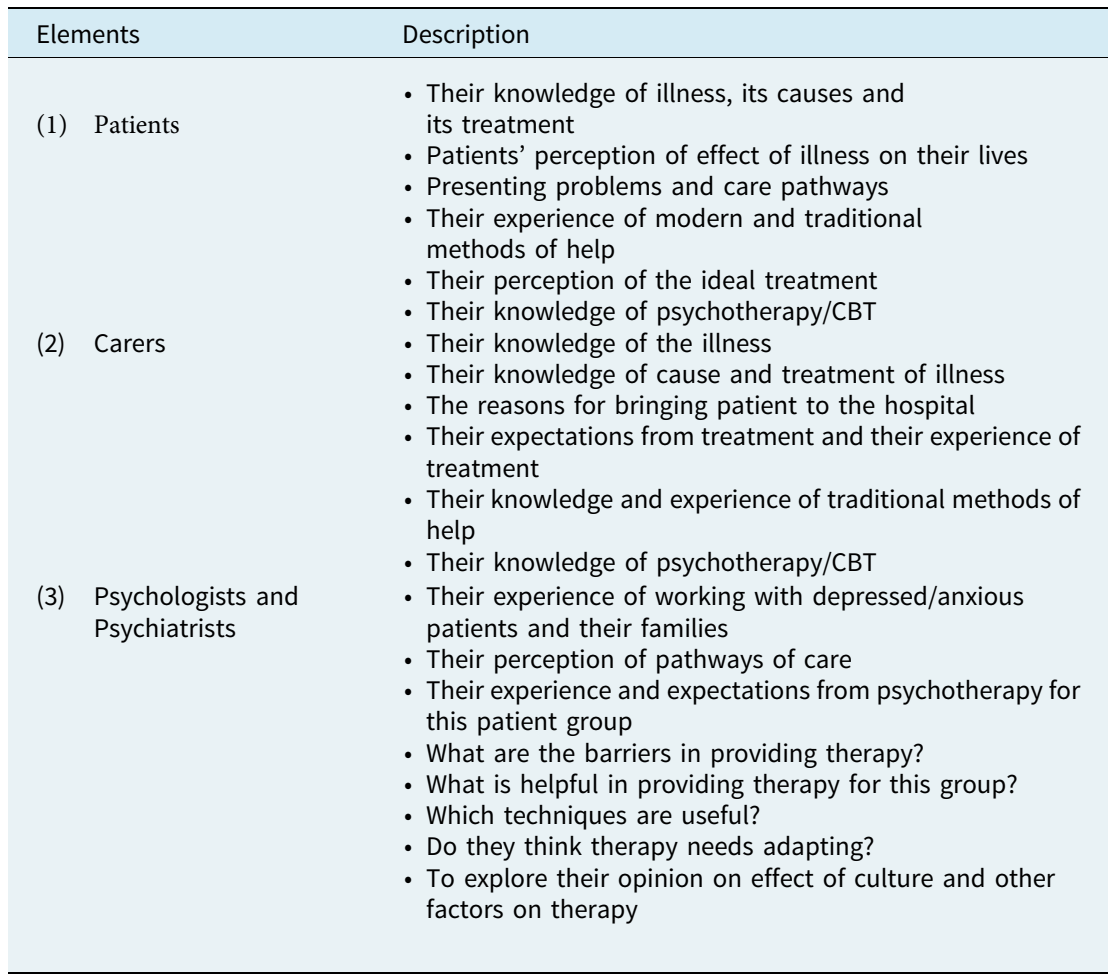

and qualitative methods. The same methodology was used in the UK to adapt CBT for psychosis. Based on that initial work, we developed a semi-structured interview guide that could be used by less experienced interviewers such as graduate students. This standardized the process and reduced the cost (Li et al., 2017; Naeem et al., 2009a; Naeem et al., 2009b, Naeem et al., 2010b; Naeem et al., 2011; Naeem et al., 2012). See Table 1 for a summary of themes explored through the interviews.

\section{Study participants}

\section{Group 1: Patients}

We interviewed patients $[n=42(\mathrm{KSA}=18, \mathrm{BAH}=24)$; gender: female $=30$, male $=12$; age: mean $=38$, range $=18-62$ ] who fulfilled our inclusion criteria, i.e. were diagnosed with depression or anxiety, fulfilling the ICD10, RDC criteria, and who were attending the outpatient clinic in one of the participating psychiatry departments. To enable us to understand their experience of mental health services, we selected patients who had at least a 1-year history of the illness. Patients with severe illness, disruptive behaviour, learning disability or severe substance misuse problems were excluded from our study.

\section{Group 2: Carers}

The caregivers $[n=11(\mathrm{KSA}=4, \mathrm{BAH}=7)$; gender: female $=7$, male $=4$; age: mean $=32$, range $=26-45$ ] attending the outpatient clinics of psychiatry departments, who were accompanying the patients, were approached and those who consented were interviewed. We selected this group because, in our previous study, we had learned that patients are commonly 
accompanied by carers and that carers are often involved in making essential decisions regarding, choice of treatment, type of health care and follow-up.

\section{Group 3: Psychologists and psychiatrists ('Psych')}

Examining the views of psychiatrists and psychologists $[n=16(\mathrm{KSA}=5, \mathrm{BAH}=11)$; gender: female $=11$, male $=5$; age: mean $=44$, range $=32-54$ ] is an essential part in understanding barriers to providing therapy and finding methods to address these barriers. We interviewed a total of nine psychologists and seven psychiatrists. We sought psychologists who preferably practised CBT. The interviewed psychologists reported that they were aware of the basic concepts of the CBT. Nearly half of the psychologists $(n=4 / 9)$ worked in the private sector.

\section{Data collection}

As it was considered essential that participants' views should be understood in the context of their profession, participation could not be completely anonymous. Therefore, participants gave written consent in the full knowledge that identifiable information would be removed from the data, but that their job titles would remain. They were given information about the study, and those who agreed were invited to the interview. No records were kept of those who refused to participate in the study, as the numbers were too small. All the interviews were conducted in a healthcare facility. The mental health professionals were interviewed at their offices. The interviews with patients and their carers were conducted in the outpatient clinics.

Each respondent participated in a semi-structured interview conducted between September 2016 and March 2017. A flexible schedule of open questions was used to guide the interviews, allowing for probing of further information and clarification where appropriate. Interviews lasted around 60 minutes and took place in the participant's place of work. Each interview was fully transcribed and checked for accuracy. Participants were made aware of their right to withdraw from the study before, during and after data collection.

Four psychiatrists conducted interviews. Interviews were conducted in Arabic and translated into English. We started transcribing interviews as soon as the process of the interviews started. Contact telephone numbers were obtained from the participants so that they could be contacted if a response needed further clarification. Access to data was limited to the research team. Field notes were taken by the researcher (H.M.S.A.) noting non-verbal communication and behaviours. Each interviewer started analysing the interviews as and when they were conducted. The interview transcripts were returned to a random sample of the professional participants for comment, verification and clarity concerning queries that arose from the analysis stage. Interviewers were provided with supervision and support through telephone and video-conferences using Skype throughout the study.

\section{Data analysis}

Data were analysed using a thematic framework method (Ritchie and Spencer, 1994) which enabled themes to be developed both from the research questions and from the narratives of research participants (Pope et al., 2000). For this study, we assumed that this is an ongoing qualitative analysis that explores themes from different cultures that can be used to culturally adapt CBT. The 'researcher (H.M.S.A.)' immersed herself in the data by carefully reading the interview scripts multiple times and identifying emerging themes and categories. Each interviewer started analysing the interviews as and when they were conducted. We followed the principle of 'emergent design' when the respondent raised the issues requiring further exploration. These issues were then tested appropriately in subsequent interviews with the 
Table 2. The framework for adaptation: the areas that adaptation process should focus on (Naeem et al., 2016)

\begin{tabular}{ll}
\hline Elements & Description \\
\hline (1) Awareness and & - Culture and related issues (culture, religion and spirituality, language and \\
preparation of therapy & communication and family-related issues) \\
& Capacity and circumstances (individual issues, e.g. gender, education, etc., and \\
& system issues, e.g. system of support and treatment, and pathways to care and \\
& help-seeking behaviour) \\
& Cognitions and beliefs (beliefs about health and illness, beliefs about treatment \\
& and treatment provider and trans-cultural variations in cognitive errors and \\
& dysfunctional beliefs) \\
& - Assessment issues (e.g. cultural identity, acculturation, language, family- \\
& related issues, cultural formulation) \\
(2) Assessment and & Engagement issues (how the clients from non-Western cultures can be engaged \\
engagement & in therapy) \\
& Barriers to therapy, culturally acceptable translation, style and focus of \\
therapy, homework, changes in therapy techniques and structural changes in \\
thechniques
\end{tabular}

participants. We also contacted participants by telephone for clarification of areas of uncertainty when the data were analysed.

Each interviewee was assigned a number for the transcription and reporting. The data were primarily descriptive, with most themes emerging in response to the interviews. We adopted an elaborate method of coding. Two teams, one located in Canada and the second based in KSA, coded data separately. This was to improve the reliability of the analysis and to ensure that the translation was working. Finally, the data were reorganized into wider themes (for example, reasons for drop-outs) and categories (for example, engagement-related issues) and written for this article.

Regular meetings between the authors were held throughout data analysis, facilitating further exploration of participants' responses, discussion of deviant cases, and agreement on recurring themes. Two of the authors (F.N. and M.A.) independently analysed the data using the thematic framework. The framework for the analysis was developed from existing literature, our previous work and from the themes that emerged from the analysis of interviews with the current study. Table 2 shows the adaptation framework used that guided our analysis.

\section{Results}

\section{Awareness and preparation of therapy}

\section{Culture and related issues}

Nearly all the professionals $(n=14 / 16)$ agreed on CBT playing an essential role in treating mental health problems and reported the need for adapting CBT to meet the local cultural and religious needs. They also talked about the translation of CBT material in Arabic taking into consideration the local idioms of distress.

'It [CBT] is very effective in anxiety-related disorders and mood-related disorder. It is one of the most supportive modalities. It is cost-effective and efficient - but it needs cultural adaptation. This is the disadvantage [of $\mathrm{CBT}$ ] that it requires lots of adaptations that need highly skilled professionals.' [Psych 7]

Another participant said, 'It [therapy] is different from the West. You have to think of religious differences, and [you] need to take into account the cultural differences, for example, the 
gender of a therapist is a sensitive issue here [as female patients might not feel comfortable with male therapists]. So, I have to make changes.' [Psych 3]

Speaking about the language and translation, one therapist said, 'This is important, language and translation - should be in Arabic. [The proper use of] language, [consideration of] culture and religious sensitivity are all important.' [Psych 12]

Most of the professionals $(n=12 / 16)$ also talked about the role played by the family in this context. Although mental health professionals described the family to be supportive in general, some of them spoke about difficulties in dealing with the families.

'The family is usually very supportive.' [Psych 4]

'We involve the family - if it is indicated. But as far as the family involvement is concernedexpressed emotions in the family are a major problem. I mean they are over-involved and overcritical.' [Psych 2]

Or, 'we have to engage family because most patients come with the family members and they want to know what is going on.' [Psych 9]

A few patients $(n=3 / 39)$ and carers $(n=2 / 11)$ also referred to the family's role, although it was mainly reported to be a negative influence. When asked about causes of their patient's illness, these carers said,

'He is weak [in character]. He is very sensitive. His family was harsh on him when he was a child.' [Carer 2]

'His problem is because of his family.' [Carer 8]

'This illness is because of my family. They cause me so much stress.' [Patient 23]

\section{Capacity and circumstances (individual and system level factors)}

The mental health professionals $(n=13 / 16)$ in our study reported the need for improved training and resources. They all agreed that it is essential to fund this treatment modality to improve services. These mental health professionals said,

'Psychotherapy is under-utilized in our culture. [The main issues are] lack of qualified people, a limited number of psychiatrists [and psychologists], no specialized centre for therapy, and, no local training for psychotherapy.' [Psych 6]

'The [health] planners [in] leading positions lack the knowledge [to realize] the importance of psychotherapy, so funding for training was not there.' [Psych 11] 'Training is a big issue; we are not [well] trained to provide different kinds of therapies.' [Psych 13]

Most of the patients $(n=30 / 39)$ and their carers $(n=12 / 16)$ were aware of the availability of CBT. Some patients had learned about CBT through social media.

'Yes - medication has helped me in the past, but I read about CBT on social media, and I think it can help me.' [Patient 15]

All mental health professionals were aware of the role played by CBT in the management of mental disorders. However, when asked for further details most $(n=10 / 16)$ did not have in-depth knowledge and instead talked about using behavioural techniques $(n=8 / 16)$. One professional talked about REBT (rational emotive behaviour therapy). These participants said, 
'I usually ask them this question ["what are their expectations?"]. It depends; some patients wish to function in life again. First, I use Roger's technique to build rapport, then Ellis's to deal with irrational thoughts, to confront thoughts and to help them improve their self-esteem. To teach them how to build their self-esteem through exposing to the real situation.' [Psych 3] 'I use CBT to help them, especially when they have anxiety or depression.' [Psych 9]

'I use exposure with my patients with OCD or phobias mainly.' [Psych 14]

The referral system withing public sector is similar in the KSA and BAH. Patients were reported to be referred through Accident \& Emergency and medical departments in the hospital, and from primary care physicians. In comparison with the private sector where the majority of patients are self-referred, a referral is required for patients to be seen in the mental health public system. The services are free for everyone in the public sector. However, one participant reported that CBT services are mainly located in bigger cities. It was also reported that the availability of CBT training is limited. The same participant suggested that there is a need to promote and even advertise it to improve access. One of the professionals highlighted the barriers within the public referral system:

'No self-referral - so that patients who are aware and motivated [but have not been referred] will not come. [They] see specialists first so pharmacotherapy will be first [treatment they receive], and psychotherapy will not be discussed with the patient unless psychiatrist knows about it and most are lacking training and knowledge in psychotherapy.' [Psych 1]

'They come from all sources. I often see patients in my private work who are referred by the friends or family or even found my name on social media.' [Psych 7]

Participants pointed to the stigma associated with mental illness. The mental health professionals did not see the involvement of spiritual or faith healers in a positive light. For example, this psychiatrist said,

'There is also a stigma about mental illness. People will come to us very late. I mean when things are overwhelming for them. Psychiatric treatment is used as the last resort; they go to millions of Sheikhs then come back to us. It is difficult [for them] to accept mental illness.' [Psych 3]

Most of the patients and the carers in our study were well educated. They were aware of psychological treatments. However, some mental health professionals $(n=3 / 16)$ expressed their concerns regarding patients' expectations from therapy. Mental health professionals when discussing the type of patients who benefit from therapy said,

'People who usually come for the psychotherapy are, [those with] mild to moderate stress, are well educated and who can wait [for therapy to start]. We also get more females than males.' [Psych 1]

It [engagement with therapy] needs a certain level of comprehension and [high level of] education.' [Psych 5]

'When they come for therapy, they think it is only chit-chat, and they use it for venting their emotions.' [Psych 12]

\section{Cognitions and beliefs (knowledge and beliefs about health and illness)}

Both patients and their carers recognized mental illness. They were also aware of their diagnoses. Some of the symptoms of the mental illness they reported were: living alone, being quiet, nervousness, shame, 'people perceiving you to be a fool', feeling jealous, selfishness and 
holding grudges. They also reported somatic concerns as their presenting problems. One carer stated his concern and said the patient might be malingering. One mental health professional, in particular, described how most patients present with physical symptoms. When asked how the illness affects their lives, some participants said;

'It made my life as if I'm swimming in a sea of honey [this means everything requires extra effort - imagine walking through a sea of honey]. My educational performance deteriorated. I am [now] totally isolated from my family.' [Patient 2]

'He is sad all the time for years. He is so passive. He hates his family. He is hopeless, isolated and does not socialize. I am the one taking care of him since marriage.' [Carer 10]

Regarding the causes of mental illness, patients $(n=32 / 42)$ and their caregivers $(n=9 / 11)$ described causes that fitted into a biopsychosocial model of illness. One patient said it was both medical and mental illness. Only three patients described spiritual factors as the cause of the disease. Some of them also expressed their views that the disease might be due to being a weak person, possibly referring to traits of a personality disorder. They said,

'It [my illness] is caused by my family, by me and by chemical imbalance.' [Patient 2]

'I am sensitive this is why [I became ill].' [Patient 3]

'It might be because of defects in neurotransmission in the brain.' [Patient 1]

'She is so sensitive, and she is weak.' [Carer 2]

'Maybe it is 25\% illness, but the rest is because of his weakness. It is called weakness of personality. It is $25 \%$ medical, $50 \%$ physical and 25\% social [in its origin].' [Carer 1]

Other participants have said that 'it might even be because of genetic or spiritual causes.'

[Patient 9]

When asked about the possible treatments, only one patient said she was not sure whether she was in the right place for the treatment (Patient 1). The same patient also said that doctors could treat the illness, but not $100 \%$. Interestingly, interviews with the mental health professionals $(n=13 / 16)$ revealed that in their opinion, the patients were most interested in medical treatments and were seeking quick fixes and full recovery. Over half of the patients $(n=23 / 39)$ and the carers $(n=6 / 11)$ reported past non-medical treatments (contact with a Sheikh, Zamzam water and reading Quranic verses). The mental health professionals $(n=2 / 16)$ expressed their frustration with the patient's expectations from the treatment:

'It is the attitude of these people - they want to feel better as soon as possible. Psychiatric help is the last resort, because the stigma is very strong, and they want to resolve the issue without trying other options.' [Psych 1]

'They want mostly pharmacotherapy, and, sometimes ECT (electro-convulsive therapy).' [Psych 1]

'They think that they can be dependent on the therapist. They expect fast improvement. They just want to lie down on a couch like in the movies.' [Psych 6]

'Their expectation is full recovery.' [Psych 1]

Patients, on the other hand, appeared to have a wish to seek treatments from a wide variety of sources. This was in line with their model of causation of illness. However, it should be emphasized that these patients were already receiving treatment and most were educated. Most of them (26/42 patients and 9/11 carers) emphasized the need for prayers and reading the Quran as additional treatments. When asked about the most appropriate treatment, they said, 
'It would be behavioural treatment, chemical treatment and CBT.' [Patient 2]

'We expect anti-depressants, CBT and social support.' [Carer 1]

'The treatment of this illness is by psychiatrist, family and the patient himself.' [Carer 1]

When asked about the role of psychotherapy and in particular about CBT, patients and their carers had a variety of opinions:

'Talking therapy - I don't believe talking can have any effect.' [Patient 3]

'I want to mention this book, 'Mind OverMood'. I did not trust CBT, but now I am using a thought diary on my 'phone on a daily basis.' [Patient 2]

'I had [a medicine] $10 \mathrm{mg}$ every day and CBT. But both were ineffective.' [Patient 1]

'I think CBT is not performed correctly. Common sense and relaxation alone could not solve life's problems.' [Caregiver 1]

Participants also talked about the role of spirituality and religion in their treatment:

'... and the person should treat himself, purify himself and use traditional remedies.'

[Carer 3]

'Other people might be able to help; like, maybe Sheikh.' [Carer 1]

'And the religious scholars with the Quran.' [Patient 14]

'I think natural ways, like breathing correctly, exercise, spiritual treatment and the medication.' [Patient 23]

\section{Assessment and engagement}

\section{Assessment issues}

The awareness that cultural and religious issues play a vital role in patients' understanding of their illness and their expectation of the healing system leads to a fair assumption that these should be taken into consideration during the assessment phase. As this mental health professional said:

'Cultural adaptation is essential for all components of therapy, starting from assessment tools.'[Psych 7]

It is also important for the therapist to explore a patient's beliefs about healing, which are underpinned by the patient's perception of aetiology, attitudes towards treatment, and healthseeking behaviour. For example, questions can be asked about what the patient/family thinks is the cause of the illness. If the therapist is not aware and respectful of the patient's beliefs about healing and his/her faith in other healing systems, the therapist might risk offending and losing the patient. It is possible that patients will not offer their views for fear of ridicule, so they could be questioned indirectly. For example, the therapist could ask what other people around the patient think are the causes of this illness. The therapist can also ask patients' opinions about spiritual and/or religious causes for symptoms (for example, whether the evil eye or sins are to blame).

\section{Engagement issues}

All the mental health professionals (16/16) in this study were aware of the importance of the therapist-patient relationship. Psychologists reported high drop-out rates among their patients. They described the following to be the reason for the dropouts:

'They don't come back because of poor compliance, long waiting time to follow-up, a lack of continuity of care, lack of patient education and stigma of psychiatry. They also stop coming 
when there is an improvement in symptoms, going to other private hospitals or faith healers.' [Psych 2]

'They sometimes stop coming after 1 or 2 sessions. The reasons include a lack of patience, patient's expectation - to get better after the first session. Some patients even fear improvement [i.e. they do not want toget better]. They are defensive and fear becoming normal, even during the inpatient treatment some remain resistant to change. Some don't want to make an effort, and it is easier to remain as they are.' [Psych 3]

The authors' experience suggests that in their semi-private clinic, patients who were referred for counselling, and later drop out of therapy, usually complain of the long wait for the follow-up appointment (1-2 months) or that therapist was not experienced enough to help with their problems.

\section{Adjustments in therapy techniques}

Barriers to therapy, culturally acceptable translation, style and focus of therapy, homework, changes in therapy techniques and structural changes in therapy

Mental health professionals described the difficulties in delivering therapy to their clients. They talked about the barriers and the need to address these barriers:

'In developing countries, the psychiatric practice started late in the region. People recognize the surgeons, not psychiatrists in this profession. So branches of psychiatry were late to develop as well. We developed general psychiatry, then children, then Consultation and Liason, and so on. And this resulted in the delay in developing psychotherapy. People have no cultural awareness of psychotherapy even in private practice.' [Psych 6]

'The CBT [practice] in this region needs a person who has an analytical mind. Some patients have difficulty in conceptualizing it, and understanding the concept behind it and require a certain level of knowledge.' [Psych 5]

'In general people do not understand the purpose of therapy. The significant barriers include institutional policies, appointment times, transportation problems, lack of improvement and poorly trained therapists.' [Psych 16]

They further talked about the barriers in delivering therapy in addition to those already described (for example, resources, training, and location of mental health services). Mental health professionals described difficulties experienced in using CBT, with some aspects of it receiving particular mention:

'Arabic patients are not interested in doing homework.' [Psych 3]

'Some of them do not have the time to come for therapy.' [Psych 2]

'In our culture, when they see a young psychologist, they will not accept advice and tasks, but if they are given medication by a young doctor, they will accept it.' [Psych 1]

'The professional does not have the knowledge or experience to implement it [CBT], especially in recognizing thoughts.' [Psych 5]

Although some of the therapists said they try to work collaboratively, they also said that patients are too dependent on them and want them to make decisions and direct them:

'In general, they depend on the doctor as the primary decision maker.' [Psych 1] 
An essential part of the interview was to explore, 'what works in therapy?'. Half $(n=8 / 16)$ of the mental health professionals mentioned the use of behavioural techniques being effective. When discussing the techniques that are easy to use and work well, they said:

'It is group therapy, problem-solving, rehabilitation of individuals, CBT and behavioural modification programs [that work well].' [Psych 3]

'These techniques are useful; cognitive confrontation, behaviourism, desensitization, exposure and problem-solving. But venting emotions is not effective.'[Psych 3]

Responding to the question of how to tackle these barriers, they emphasized the importance of psychoeducation and dealing with the families effectively:

'We educate the families.' [Psych 3]

'The family is involved from the beginning. I invite the family to talk about the patient and discuss the other treatment options. And to convince the patient to accept treatment, explaining tasks can be helpful.' [Psych 1]

They also suggested some structural changes and ideas that can make CBT more accessible and acceptable to the patients from this background:

'... to advertise more about psychotherapy in ways that are more accepted in our culture; to conduct it in social clubs, community centres and not only in psychology centres, or different settings, and, in schools. We can develop very short versions and shorter sessions to improve engagement. A long session can lead to boredom, for example, sometimes the sessions with a patient can last for 2 hours.' [Psych 1]

\section{Discussion}

The results of this study are consistent with previous literature, i.e. CBT may need to be modified for clients from non-Western cultural backgrounds (Hays and Iwamasa, 2006; Iwamasa, 1993; Naeem et al., 2010b). These findings also confirm our previous results, i.e. the role of awareness of cultural factors, and their implementation in assessment for, engagement in, and adjustments in techniques of therapy. We were able to use the methodology adopted from our previous work to explore stakeholder's opinions regarding the cultural adaptation of CBT.

The health professionals interviewed in our study emphasized the need to be aware of cultural, religious and other factors while providing therapy. Participants observed that several resources (books, outcome measures, etc.) have already been translated into the Arabic language, but quite often conceptual meanings are lost in the process of translation. Use of the classic Arabic terms can cause a communication barrier for the therapist and clients. This highlights the need for culturally sensitive translation of therapy material. Our previous work in Pakistan highlighted this problem, and we adopted a 'name the title' technique to translate specific terminology (Naeem et al., 2009b) instead of literal translations (in this technique a concept is explained to laypersons and they are asked to give it a title, for example, they are given examples of black and white thinking with explanation, and are asked what do they call this type of thinking in their language). Therapists conducting therapy should inquire about the role of religion in the patient's life and include it in the treatment plan when indicated. Knowledge about religion can be beneficial to the therapist in addressing specific misconceptions and distorted thinking in almost all disorders. 
Some of the professionals we interviewed raised concerns regarding the role of the family. There is evidence from the literature that involving the family in therapy can have a positive influence on the therapy outcome (for example, psycho-education and providing a rationale for treatment may result in better compliance, and may reduce patient's distress; similarly, involving the family can play a vital role in improved home work compliance) (Naeem et al., 2016). This is an area that needs further exploration. Social structures are changing in non-Western cultures. This also means a change in family structure and their role. It is also possible that health professionals trained under a Western model will have a less favourable view of the part of the family in therapy.

Participants in our interviews also discussed the broader role the system plays. The referral system in KSA and BAH might be different from that in the UK, Pakistan and China. They mentioned a lack of training opportunities in therapy. Psychotherapy training was mandated in 2012 by the Saudi Council of Health Specialties to be part of Psychiatry residency training where residents have to have an exposure to some cases, of which two shall be in CBT. As of the date of writing this article, there are no mechanisms in place to ensure compliance of the different training programmes with this recommendation. Also, the availability of supervision and supervisors who have experience in CBT is quite variable. They highlighted the role stigma plays as a barrier to help-seeking. We believe, however, that its degree depends on the perceived causes of the illness. Therefore, depending on what is the perception of illness the treatment sought will follow accordingly. In Islam, finding treatment when you are ill is mandatory, and healing requires addressing health from spiritual, medical and psychological perspectives. As per interviewed professionals, going to a Sheikh is much more comfortable and culturally accepted for many patients in the region than seeing a psychiatrist. An additional barrier was their understanding of the causes of the illness. This is in line with our previous work, in which $40 \%$ of respondents of a survey believed mental diseases to be due to a chemical imbalance, $30 \%$ thought it to be due to weak faith, and personality, while $30 \%$ thought it is due to genetics (H.M.S.A., unpublished data). It is therefore understandable that patients consult healers from a variety of backgrounds.

Therapists discussed the barriers to therapy and adjustments they make in treatment. They particularly highlighted the difficulties in completing homework which could reflect a therapist's inadequate training and/or expereince The first two authors, who were trained in North America, have observed that the overall proportion of patients, who do not finish their homework locally, is similar to that in the West. Providing a rationale for therapy, involving family, and keeping it simple helps with the completion of homework. These authors have noticed, during supervision of trainee CBT therapists, that they tend to give patients multiple scales and 8-column thought records to complete in the first few sessions, without adequate explanation or preparatory work. Similarly, people in KSA and BAH are likely to present with somatic symptoms more than affective symptoms, which newly trained therapists have to keep in mind when tackling difficulty in eliciting emotions.

This work shows that CBT might need minor adjustments for use in Arab countries such as KSA and Bahrain. The issues raised can be easily incorporated into CBT to inform 'culturally sensitive assessment and formulation'. Being aware of the cultural factors might also help to improve engagement. Findings from this study are consistent with the guidelines that were previously described for delivering culturally adapted CBT (Naeem et al., 2010a; Naeem et al., 2010b; Naeem et al., 2016). We are observing specific recurrent themes in our adaptation work that appear to apply more generally than in one country or culture. These include being aware of cultural factors in therapy, language and translation of English concepts, the role of the family, understanding care pathways and the role of religion in illness belief models. Similarly, only minor adjustments in therapy techniques were suggested. 
To our knowledge, this is the first systematic attempt to explore the need to culturally adapt CBT for anxiety and depression for this population. However, there is some evidence in the literature that confirms these findings. For example, the role of family, language and consideration of wider cultural and religious factors have previously been described to be vital in this context (Abudabbeh and Hays, 2006; Dwairy, 2006). A recently reported RCT of internet-delivered CBT for an Australian Arab population emphasized the role of cultural sensitivity in the translation of therapy manuals (Kayrouz et al., 2015). A systematic review of mental health interventions in Middle Eastern Arab countries identified cultural context, engagement with the services, the role of religious beliefs and language and communication to be the major barriers to their acceptance (Gearing et al., 2013). Similarly, a qualitative study exploring the explanatory models (EMs) of mental illness among future Emirati mental health practitioners highlighted the influence of cultural traits on the formulation of EMs, the importance of family in the Emirati society and the impact of the UAE socio-economic and religious reality in forming the participants' beliefs regarding mental illness (Petkari, 2015).There is a need to further this work to validate the generalization of our findings.

\section{Implications for therapy and research}

The information gathered in this study can be easily used to inform culturally sensitive assessment and formulation to engage clients as well as suggest minor adjustments of CBT process and/or techniques. An understanding and grasp of the cultural factors will enhance the process of patient engagement with therapy and care, thus reducing drop-outs and improving therapy outcomes. Future studies should develop and propose culturally adapted CBT (CaCBT) using the guidelines developed from this study, in these settings, then focus on testing the efficacy of such adaptations. There may also be a need to adjust CBT for disorders other than depression and anxiety. There may be a need to develop culturally sensitive self-help interventions that can address larger numbers with low severity of problems. Finally, there should be studies to compare CaCBT with the standard CBT.

\section{Limitations}

Although KSA and BAH are similar in their culture, there might be subtle differences that this research might have failed to capture. The mental health professionals interviewed in this project were practicing in large teaching hospitals or big cities and patients, and their carers were also mostly city dwellers. This may put some limits on the generalizability of the findings, although including three different groups of participants in the study would have addressed this issue at least partially. Interviews have been criticized over their validity, but in our experience, these are useful tools in the process of adaptation of CBT as we have shown in our other work cited in this manuscript. An additional mitigating factor that gives us confidence in the value of interviews is the similarity in the results to our previous work in Pakistan, Morocco, China and the Middle East, and with ethnic minority clients in the United Kingdom. Another limitation may have to do with the actual impact of adapting CBT without changes to the systems of care within which it is delivered. Adapting CBT alone without improving access to it may not result in substantially improved care for patients. Given that wide variations exist in the health systems of non-Western countries, cultural adaptations will not be enough until and unless access to CBT is improved across the board in these countries.

Acknowledgments. The authors would like to extend their appreciation to the patients, caregivers and mental health professionals who participated in this research, and to Dr Yasser Ad-Dab'bagh, Executive Director, Research Executive Administration, King Khalid Medical City (REA-KKMC) for his revision and proofreading of our paper.

Conflicts of interest. The authors declare no conflicts of interest. 
Ethical statements. The authors have abided by the Ethical Principles of Psychologists and Code of Conduct as set out by the APA. IRB approval was obtained from King Fahd Hospital of the University (KFHU), Imam Abdulrahman Bin Faisal University in AlKhobar, KSA, and Salmaniyah Psychiatric Hospital in Manama, BAH (REC/PSY012015/075).

Financial support. This study was not funded by any party.

Key practice points

(1) CBT can be systematically adapted using a series of steps and involving stakeholders.

(2) The focus of cultural adaptation of CBT should focus on three major areas, i.e. awareness of culture and religion, assessment and engagement, and adjustments in therapy.

(3) Lessons learned from this study confirm our previous findings in cultural adaptation of CBT.

\section{Further reading}

Abudabbeh, N., \& Hays, P. A. (2006). Cognitive-behavioral therapy with people of Arab heritage. In P. A. Hays \& G. Y. Iwamasa (eds), Culturally Responsive Cognitive-Behavioral Therapy: Assessment, Practice, and Supervision (pp. 141-159). Washington, DC, USA: American Psychological Association.

Naeem, F., Phiri, P., Nasar, A., Munshi, T., Ayub, M., \& Rathod, S. (2016). An evidence-based framework for cultural adaptation of cognitive behaviour therapy: process, methodology and foci of adaptation. World Cultural Psychiatry Research Review, 11, 67-70.

\section{References}

Algahtani, H., Buraik, Y., \& Ad-Dab’bagh, Y. (2017). Psychotherapy in Saudi Arabia: its history and cultural context. Journal of Contemporary Psychotherapy, 47, 105. https://doi.org/10.1007/s10879-016-9347-2.

Abudabbeh, N., \& Hays, P. A. (2006). Cognitive-behavioral therapy with people of Arab heritage. In P. A. Hays \& G. Y. Iwamasa (eds), Culturally Responsive Cognitive-Behavioral Therapy: Assessment, Practice, and Supervision (pp. 141-159). Washington, DC, USA: American Psychological Association. https://doi.org/10.1037/11433-006.

Bernal, G., Bonilla, J., \& Bellido, C. (1995). Ecological validity and cultural sensitivity for outcome research: issues for the cultural adaptation and development of psychosocial treatments with Hispanics. Journal of Abnormal Child Psychology, 23, 67-82. https://doi.org/10.1007/BF01447045

Bernal, G., Jiménez-Chafey, M. I., \& Domenech Rodríguez, M. M. (2009). Cultural adaptation of treatments: a resource for considering culture in evidence-based practice. Professional Psychology: Research and Practice, 40, 361-368. https://doi.org/ 10.1037/a0016401

Creswell, J. W. (2009). Research Design: Qualitative, Quantitative, and Mixed Methods Approaches, p. 297. Sage Publications.

Domenech Rodríguez, M., Baumann, A., \& Schwartz, A. (2010). Cultural adaptation of an evidence based intervention: from theory to practice in a Latino/a community context. American Journal of Community Psychology, 47, 170-186.

Draine, J., \& Solomon, P. (1994). Explaining attitudes toward medication compliance among a seriously mentally ill population. Journal of Nervous and Mental Disease, 182, 50-54.

Dwairy, M. (2006). Counseling and Psychotherapy with Arabs and Muslims: A Culturally Sensitive Approach. New York, NY, USA: Teachers College Press.

Gaytandjieva, A., \& Bontcheva, I. (2013). Why do we fail to adapt to a different culture? A development of a therapeutic approach. International Journal of Psychiatry, 17, 43-58. https://www.researchgate.net/publication/259620279_Why_ do_we_fail_to_adapt_to_a_different_culture_A_development_of_a_therapeutic_approach.

Gearing, R. E., Schwalbe, C. S., MacKenzie, M. J., Brewer, K. B., Ibrahim, R. W., Olimat, H. S., \& Al-Krenawi, A. (2013). Adaptation and translation of mental health interventions in Middle Eastern Arab countries: a systematic review of barriers to and strategies for effective treatment implementation. International Journal of Social Psychiatry, 59, 671-681. https://doi. org/10.1177/0020764012452349.

Hays, P. A., \& Iwamasa, G. (2006). Culturally Responsive Cognitive-Behavioral Therapy: Assessment, Practice, and Supervision. American Psychological Association.

Hwang, W.-C. (2006). The psychotherapy adaptation and modification framework: application to Asian Americans. American Psychologist, 61, 702-715. https://doi.org/10.1037/0003-066X.61.7.702.

Hwang, W.-C., Myers, H., Chiu, E., Mak, E., Butner, J., Fujimoto, K., \& Miranda, J. (2015). Culturally adapted cognitive behavioral therapy for depressed Chinese Americans: a randomized controlled trial. Psychiatric Services (Washington, D.C.), 66, 1035-1042. https://doi.org/10.1176/appi.ps.201400358 
Iwamasa, G. Y. (1993). Asian Americans and cognitive behavior therapy. The Behaviour Therapist, 16, $233-235$.

Joel, D., Sathyaseelan, M., Jayakaran, R., Vijayakumar, C., Muthurathnam, S., \& Jacob, K. S. (2003). Explanatory models of psychosis among community health workers in South India. Acta Psychiatrica Scandinavica, 108, 66-69.

Kayrouz, R., Dear, B. F., Johnston, L., Gandy, M., Fogliati, V. J., Sheehan, J., \& Titov, N. (2015). A feasibility open trial of guided Internet-delivered cognitive behavioural therapy for anxiety and depression amongst Arab Australians. Internet Interventions - The Application of Information Technology in Mental and Behavioural, 2, 32-38. https://doi.org/10. 1016/j.invent.2014.12.001.

Kayrouz, R., Dear, B.F., Kayrouz, B., Karin, E., Gandy, M., \& Titov, N. (2018) Meta-analysis of the efficacy and acceptability of cognitive-behavioural therapy for Arab adult populations experiencing anxiety, depression or post-traumatic stress disorder. Cognitive Behaviour Therapy, 47, 412-430. doi: 10.1080/16506073.2018.1445124. Epub 2018 May 1.

Kleinman, A. (1980). Patients and Healers in the Context of Culture: An Exploration of the Borderland Between Anthropology, Medicine, and Psychiatry. Berkeley: University of California Press.

Kohn, L. P., Oden, T., Muñoz, R. F., Robinson, A., \& Leavitt, D. (2002). Brief report: adapted cognitive behavioral group therapy for depressed low-income African American women. Community Mental Health Journal, 38, 497-504. https://doi. org/10.1023/A:1020884202677

Kvale, S. (1996). Interviews: An Introduction to Qualitative Research Interviewing, p. 352. Sage Publications.

Kvale, S., \& Brinkmann, S. (2009). Interviews: Learning the Craft of Qualitative Research Interviewing (2nd edn). Thousand Oaks, CA, USA: Sage Publications, Inc.

Lazare, A., Eisenthal, S., \& Wasserman, L. (1975). The customer approach to patienthood: attending to patient requests in a walk-in clinic. Archives of General Psychiatry, 32, 553-558. https://doi.org/10.1001/archpsyc.1975.01760230019001

Li, W., Zhang, L., Luo, X., Liu, B., Liu, Z., Lin, F., \& Naeem, F. (2017). A qualitative study to explore views of patients, carers and mental health professionals to inform cultural adaptation of CBT for psychosis (CBTp) in China. BMC Psychiatry, 17. https://doi.org/10.1186/s12888-017-1290-6

Littlewood, R. (1990). From categories to contexts: a decade of the 'new cross-cultural psychiatry'. British Journal of Psychiatry, 156, 308-327. https://doi.org/10.1192/bjp.156.3.308

Morse, J. M., \& Field, P. A. (1996). Nursing Research: The Application of Qualitative Approaches, p. 208. Stanley Thornes Ltd.

Naeem, F., Gobbi, M., Ayub, M., Kingdon, D., \& others. (2010a). Psychologists experience of cognitive behaviour therapy in a developing country: a qualitative study from Pakistan. International Journal of Mental Health Systems, 4 (2).

Naeem, F., Ayub, M., Gobbi, M., \& Kingdon, D. (2009a). Development of Southampton adaptation framework for CBT (SAF-CBT): a framework for adaptation of CBT in non-western culture. Journal of Pakistan Pyschiatric Society, 6, 79-84.

Naeem, F., Gobbi, M., Ayub, M., \& Kingdon, D. (2009b). University students' views about compatibility of cognitive behaviour therapy (CBT) with their personal, social and religious values (a study from Pakistan). Mental Health, Religion \& Culture, 12, 847-855. https://doi.org/10.1080/13674670903115226

Naeem, F., Habib, N., Gul, M., Khalid, M., Saeed, S., Farooq, S., Kingdon, D. (2014). A qualitative study to explore patients', carers' and health professionals' views to culturally adapt CBT for psychosis (CBTp) in Pakistan. Behavioural and Cognitive Psychotherapy, 44, 43-55. https://doi.org/10.1017/S1352465814000332

Naeem, F., Phiri, P., Nasar, A., Munshi, T., Ayub, M., \& Rathod, S. (2016). An evidence-based framework for cultural adaptation of cognitive behaviour therapy: process, methodology and foci of adaptation. World Cultural Psychiatry Research Review, 11, 67-70.

Naeem, F., Phiri, P., Rathod, S., \& Kingdon, D. (2010b). Using CBT with diverse patients. Working with South Asian Muslims. In The Oxford Guide to Surviving as a CBT Therapist (p. 55). Oxford: Oxford Universirty Press. Retrieved from: http://eml.manchester.ac.uk/lib/MEDN62031/MEDN62031_4624.pdf

Naeem, F., Waheed, W., Gobbi, M., Ayub, M., \& Kingdon, D. (2011). Preliminary evaluation of culturally sensitive CBT for depression in Pakistan: findings from Developing Culturally-sensitive CBT Project (DCCP). Behavioural and Cognitive Psychotherapy, 39, 165-173. https://doi.org/10.1017/S1352465810000822

Petkari, E. (2015). Explanatory models of mental illness: a qualitative study with Emirati future mental health practitioners. Mental Health, Religion \& Culture, 18, 738-752. https://doi.org/10.1080/13674676.2015.1091447

Pope, C., Ziebland, S., \& Mays, N. (2000). Analyzing qualitative data. BMJ, 320, 114-116. https://doi.org/10.1136/bmj.320. 7227.114

Rathod, S., Gega, L., Degnan, A., Pikard, J., Khan, T., Hussain, N., Munshi, T., \& Naeem, F. (2018). The status of culturally adapted mental health interventions: a practice-focused review of meta-analyses. Neuropsychiatric Disease and Treatment, 14, 165-178. https://doi.org/10.2147/NDT.S138430

Rhermoul, F.-Z. E., Naeem, F., Kingdon, D., Hansen, L., \& Toufiq, J. (2017). A qualitative study to explore views of patients, carers and mental health professionals' views on depression in Moroccan women. International Journal of Culture and Mental Health, 11, 178-193. https://doi.org/10.1080/17542863.2017.1355397

Ritchie, J., \& Spencer, L. (1994). Qualitative data analysis for applied policy research. In Bryman, A. \& Burgess, R. (eds), Analyzing Qualitative Data (pp. 173-194). London, UK: Routledge. Retrieved from: https://doi.org/10.4324/ 9780203413081_chapter_9 
Scorzelli, J. F., \& Reinke-Scorzelli, M. (1994). Cultural sensitivity and cognitive therapy in India. The Counseling Psychologist, 22, 603-610. https://doi.org/10.1177/0011000094224006

Tseng, W.-S. (2004). Culture and psychotherapy: Asian perspectives. Journal of Mental Health, 13, 151-161. https://doi.org/ 10.1080/09638230410001669282

Weiss, M. (1997). Explanatory Model Interview Catalogue (EMIC): framework for comparative study of illness. Transcultural Psychiatry, 34, 235-263. https://doi.org/10.1177/136346159703400204

Cite this article: Algahtani HMS, Almulhim A, AlNajjar FA, Ali MK, Irfan M, Ayub M, and Naeem F. Cultural adaptation of cognitive behavioural therapy (CBT) for patients with depression and anxiety in Saudi Arabia and Bahrain: a qualitative study exploring views of patients, carers, and mental health professionals. The Cognitive Behaviour Therapist. https://doi.org/ $10.1017 /$ S1754470X1900028X 